Article

\title{
Research on the Aerodynamic Characteristics of Tractor-Trailers with a Parametric Cab Design
}

\author{
Jing Peng, Tie Wang *, Tiantian Yang, Xiuquan Sun and Guoxing Li \\ College of Mechanical Engineering, Tai Yuan University of Technology, Taiyuan 030024, China; \\ pengjing518@126.com (J.P.); ytt1214@163.com (T.Y.); sxquan62@163.com (X.S.); liguoxing@tyut.edu.cn (G.L.) \\ * Correspondence: wangtie@tyut.edu.cn; Tel.: +86-139-3453-0090
}

Received: 7 February 2018; Accepted: 10 May 2018; Published: 15 May 2018

\begin{abstract}
As tractor-trailer ownership has increased year-by-year, corresponding energy consumption and environmental issues have gradually become a heated issue. Approximately 45 percent of the total aerodynamic drag of tractor-trailers is attributed to the flow at the front surface of the cab, and the gap between the cab and trailer. Therefore, this study has taken a new approach to designing the shape of the cab inspired by the external forebody of the cheetah. A parametric design protruding cab was devised in this study; the length of the protruding part and the angle between this protruding part and the A pillar were two design variables. Computational fluid dynamics simulation was conducted to investigate the drag reduction effects of these new cab styling designs, and the proposed cab reduced the drag by a maximum of $8.49 \%$. The flow characteristics around the whole body of the tractor-trailer baseline model and the parametric cab design vehicle model were analyzed using the velocity streamline graph to illuminate the change of the flow field and the drag-reduction mechanism of the proposed design. A vortex formed above the protruding part of the cab and it acted as a 'vortex cushion' to accelerate the speed of the air flow; accordingly, the positive and negative pressure distribution on the front surface of the cab and trailer also changed. The new parametric design has provided the possibility to adjust the forebody of the cab to an aerodynamically optimized shape, and these findings have offered useful information for the development of a new design method of the tractor-trailer to reduce aerodynamic drag and improve fuel economy.
\end{abstract}

Keywords: tractor-trailer; parametric design; drag reduction; vortex cushion effect

\section{Introduction}

In 2016, the total length of Chinese expressways exceeded 130,000 km, ranking first in the world, and another $5000 \mathrm{~km}$ of highways was added in 2017. Road transportation accounts for nearly $77 \%$ of the total volume of transported goods, and tractor-trailers played an important role in this. According to the auto industry production and marketing data released by CAMM (China association of automobile manufacturers), in 2016, the production and sales of commercial vehicles totaled 370 million and 365 million, respectively, and the numbers of tractor-trailers were 74.14 million and 73.29 million, which increased $38.29 \%$ and $33.08 \%$ year-by-year. The increase in vehicle ownership has resulted in greater energy consumption. For a tractor-trailer, the purchase price is fixed and disposable, while the operational cost is long term. In the course of its operation, the aerodynamic drag of the body directly affects performance and fuel economy, and the fuel consumption caused by the air resistance accounts for most of its transportation costs. Therefore, it is necessary to study methods of drag reduction for tractor-trailers to reduce fuel consumption.

The aerodynamic characteristics of passenger vehicles have been extensively investigated in the past two decades [1-3]. In recent years, the aerodynamic performance and geometry optimization of tractor-trailers have received much more attention, and many studies have been conducted to 
reduce their aerodynamic drag [4-7]. At present, by means of wind tunnel tests and numerical simulations, researchers have found that four critical parts of these vehicles make up the major parts of the aerodynamic drag of a tractor-trailer, as shown in Figure 1; these are the forebody of the tractor, the gap between the cab and the trailer, the underhood plus the wheels, and the rear of the trailer [8,9]. For a tractor-trailer driving on a highway, the front part of the vehicle causes approximately $45 \%$ of the total drag force; the other contributing factors include the rear wake of the trailer (25\%) and the underbody flow (30\%) [10]. Corresponding research was carried out at different positions, and various kinds of drag-reducing devices have been introduced to effectively decrease the aerodynamic drag of tractor-trailers, including their cab roofs or gap fairings [11-14], their side skirts [15], their base flaps [16], and their boat tails [17,18]. Some studies focused on their aerodynamic characteristics, using a combination of various devices and interactions with the actual complex shape of tractor-trailers $[19,20]$.

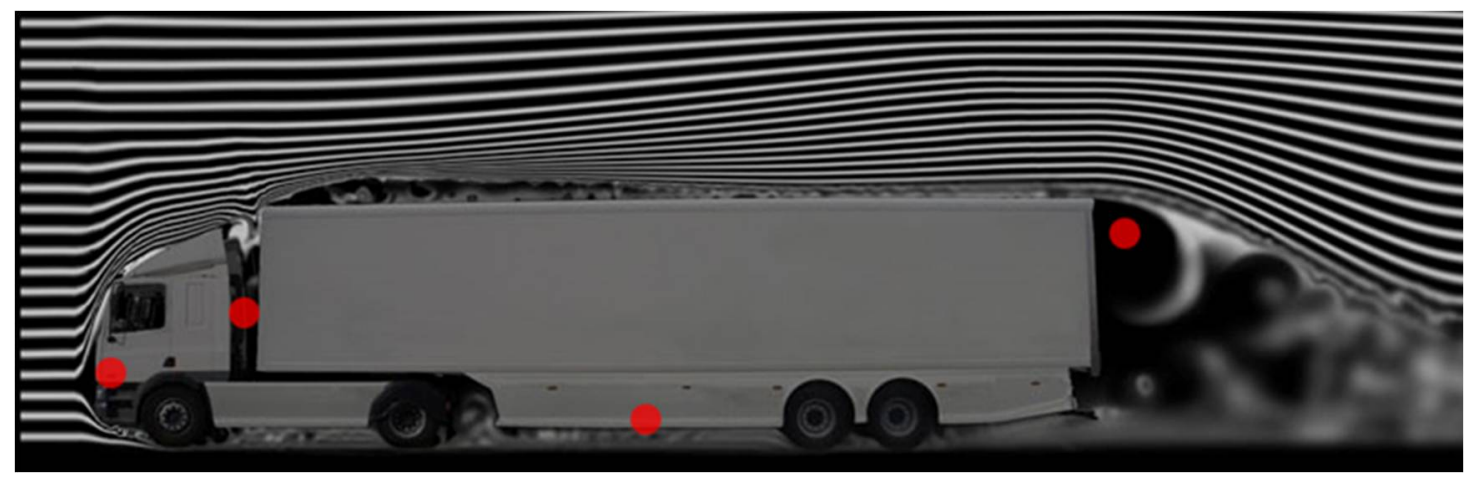

Figure 1. Four key locations that affect the aerodynamic characteristics of the combined vehicle.

Previous studies mostly focused on performance optimizations using aerodynamic drag reduction devices. Little analysis has been conducted on aerodynamic drag reduction effects based on the adjustment of the tractor-trailer shape itself, although this method will only involve a small additional cost, but could avoid the matching problem between the pneumatic accessories and the vehicle. Mario Hirz (2013) designed a variable outer contour of the trailer to adjust the rear end; the curved top surface of the trailer was most obviously changed, and, compared to the initial vehicle the aerodynamic drag, was clearly reduced [21]. Furthermore, except for the existing drag reduction methods, commercial vehicle brands, such as SCANIA tractor-trailer (SCANIA, Malmo, Sweden), VOLVO tractor-trailer (Volvo Trucks, Göteborg, Sweden), or TESLA Semi electric tractor-trailer (TESLA, Palo Alto, CA, USA), have begun to explore new styling designs of the cab to reduce the aerodynamic drag force of tractor-trailers.

The way to solve the engineering problems can be obtained from living creatures found in nature, because they possess optimal morphological structures that have been adapted to their environments. Marine and terrestrial animals have often been used as a reference for designers to study when developing new transportation styling design or methods for aerodynamic drag reduction. For example, a concept vehicle design developed by Mercedes Benz originated from the bionic design of a Boxfish found in the ocean. Dongri Kim (2015) designed the automatic moving deflector (AMD), which was inspired by the movement of secondary feathers on a bird's wing suction surface. In this study, several AMDs of different sizes and materials were tested by measuring the drag force on the Ahmed body, and displayed drag reductions up to 19\% [22]. Jeong Jae Kim (2017) was enlightened by the forehead shape of the sea lions and designed two cab-roof fairing (CDF) models. It was seen from the wind tunnel test that the redesign CDFS reduced the regions of separated shear flow and turbulent kinetic energy level on the side surfaces of the tractor-trailer model [23].

A number of terrestrial animals, like the cheetah, can run at a high speed of up to $90 \mathrm{~km} / \mathrm{h}$; this value is close to the speed that tractor-trailers travel on Chinese expressways. Meanwhile, cheetahs 
and the tractor-trailers all run in the air at similar speeds, and both of them display similar values of the Reynolds number $\left(R_{e}=\sim 10^{6}\right)$. Cheetahs can considerably reduce their drag because of their streamlined body, which consists of a protruding head and smooth body lines. Thus, inspired by the forehead shape of the cheetah, a new protruding cab with parametric design was devised in this study for the drag reduction of tractor-trailers.

According to the requirements of the cab, the 3D forehead shape of the cheetah was properly adjusted to form a slightly protruding forward shape, and its dimensions that were formulated refer to the tractor-trailer baseline model. The objective of the study was to investigate the flow field over the tractor-trailer model with a new cab shape design, and to demonstrate and describe the difference between the flow field in a direct comparison. The analysis in this paper has focused on the relative differences in the flow over the cab, the gap between the cab and trailer, and the rear part of the models caused by the different cab shapes and dimensions, as well as the consequent changes in the size and distribution of the air pressure. We also investigated the drag reduction effects of several parameters of the cab design, including the length of the protruding part and the angle between this protruding part and the A pillar.

\section{Research Approach}

The aerodynamic characteristics of tractor-trailer could be obtained by wind tunnel test and numerical simulation, and the wind tunnel test could be divided into quantitative or qualitative experiments. Aerodynamic force measurement is a commonly used quantitative experiment that includes pressure measurement and velocity measurement. Qualitative experiments are always used to display the flow field, and PIV measurement is a common method [24,25].

The aerodynamic simulation of a tractor-trailer is also a complex phenomenon; many factors determine the accuracy of this process, such as the geometric model, mesh generation, and numerical calculation scheme. In the next section, the main stages of the simulation that was performed have been described.

In this paper, the aerodynamic force measurement experiment is conducted, and the main purpose of the wind tunnel test is to validate the correctness of the numerical simulation by the comparison of $\boldsymbol{C}_{\boldsymbol{d}}$. Then, through numerical simulation, we aim to study the changes in the aerodynamic characteristics of tractor-trailer caused by the parametric design cab.

\subsection{The Model}

\subsubsection{Tractor-Trailer Baseline Model}

Several tractor-trailer flow studies have been performed using bluff or generic vehicle bodies. These investigations showed universal trends, and the research findings have general applications. However, these simplified vehicle models were somewhat different from the real product in their detailed shape and loading conditions; therefore, the impact of different modeling details on the flow structure cannot be fully realized. The baseline model studied for this paper was based on an actual flat head tractor-trailer commonly used in China.

Considering the dimensions of the wind tunnel test section and the calculation amount of the numerical simulation, the geometric scale of the baseline vehicle model was set as 1:15, and the geometry and dimensions of the tractor-trailer baseline model used have been shown in Table 1 . Due to the constraints of physical model dimensions and the effects of computational accuracy, many vehicle body details such as front headlights, wipers, and door handles were simplified after careful consideration. In addition, the emphasis of this paper was to study the outflow of the tractor-trailer, in the same way as with other research findings [26-28], the baseline model was designed with a smooth underbody and without openings for cooling airflow, and the model used in wind tunnel test and numerical simulation is shown in Figure 2. 
Table 1. Dimensions of the tractor-trailer baseline model.

\begin{tabular}{cc}
\hline Baseline Model & Geometric Parameters (mm) \\
\hline Length (L) & 1014 \\
Width (W) & 187 \\
Height (H) & 281 \\
Wheelbase (Wb) & 171 \\
Track (T) & 116 \\
Front Overhang (FO) & 99 \\
Rear Overhang (RO) & 100 \\
\hline
\end{tabular}
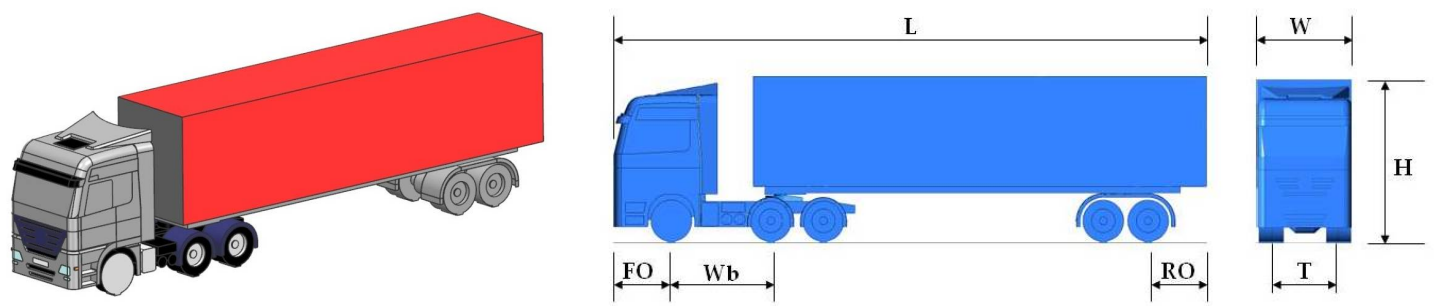

Figure 2. Schematic of the 1:15 tractor-trailer baseline model. FO was Front Overhang, RO was Rear Overhang, Wb was Wheelbase.

\subsubsection{Parametric Cab Design Model}

In this paper, the aim of the cab styling design was to imitate the protruding head shape of a cheetah and change the airflow structure in the front of the cab. Based the design of the flat head cab, the engine compartment of the new cab was designed to protrude forward to form a similar 'cab nose'.

The length of the protruding part ( $\mathrm{L}$ as shown in Figure $3 \mathrm{~b}$ ) was set as one of the modeling design variables. According to the Chinese automobile industry standard, the length of the whole tractor-trailer cannot be more than $2 \times 10^{5} \mathrm{~mm}$, and the length of the baseline cab in this study was $2200 \mathrm{~mm}$. Thus, $\mathrm{L}$ of the new cab was set to $400 \mathrm{~mm}, 500 \mathrm{~mm}$, and $600 \mathrm{~mm}$. These increased lengths would not greatly affect the loading of the rear trailer by the constraints of the standard.

The angle between the A pillar and the horizontal plane is a key factor that affects the aerodynamic characteristics of the vehicle [29]; for the initial cab, this angle was 70 degrees. Therefore, the angle between this protruding part and A pillar was another modeling design variable ( $\theta$ as shown in Figure $3 \mathrm{~b}$ ), and $\theta$ was set to 120 degrees, 125 degrees, 130 degrees, and 135 degrees, respectively.

Based on two design variables and their range of values, twelve cab design schemes were obtained; Figure $3 \mathrm{c}$ shows three of these cab designs. According to the requirements of the industry standard and the feasibility of the design, these two modeling design variables cannot grow or increase optionally; otherwise, they will form a singular shape.

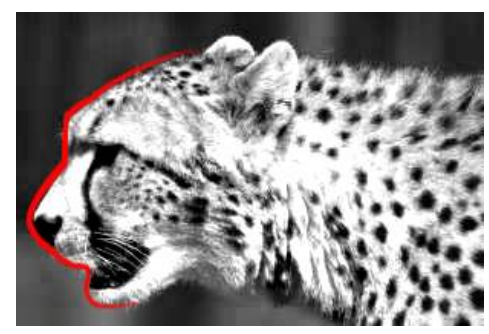

(a)

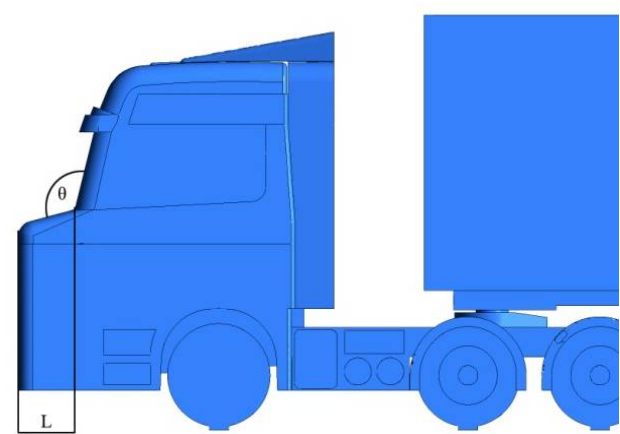

(b)

Figure 3. Cont. 


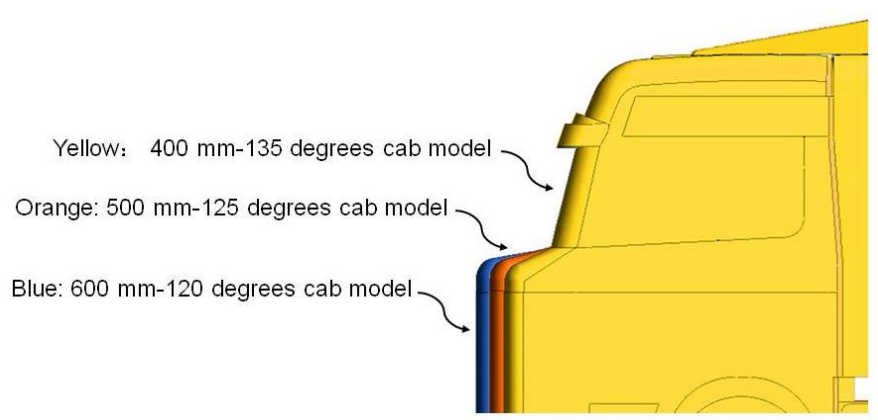

(c)

Figure 3. (a) External forehead shape of a cheetah; (b) two modeling design variables of new cab design, $\mathrm{L}$ (400 mm, $500 \mathrm{~mm}$, and $600 \mathrm{~mm}$ ), $\theta$ (120 degrees, 125 degrees, 130 degrees, and 135 degrees); and (c) partial cab design schemes.

\subsection{CFD Simulation Set up}

Both the baseline model and the tractor-trailer models with parametric design cabs were drawn using UG (10.0, Siemens PLM Software Company, Plano, TX, USA, 2014), and imported into ICEM (16.0, ANSYS Company, Canonsburg, PA, USA, 2015) to produce dividing grids. Taken the baseline model as an example and shown in Figure 4, a box was created around the models to define the computation domain. The established principle of the computational domain in CFD is the boundary, which does not affect the flow field around the vehicle; meanwhile, the range is be minimized [30-32]. In this paper, the clearance between the inlet and the front of the cab was $3 \mathrm{~L}$, and the clearance between the outlet and the back of the trailer was $7 \mathrm{~L}$, in which $\mathrm{L}$ was the length of the baseline model; a clearance of $5 \mathrm{~W}$ was used between the side of the box and the model on both sides, and a clearance of $4 \mathrm{H}$ was used between the top of the box and the model, in which $\mathrm{W}$ was the width of the model and the $\mathrm{H}$ was the height. The block ratio of this study was $2.27 \%$, it satisfied the experiment requirements. For every parametric cab design tractor-trailer model, one difference was the increased length of protruding part of cab; thus, the length of computing domain increased with the cab length, and the clearance between the inlet and the front of the parametric designed cab kept $3 \mathrm{~L}$; the other parameters remained.

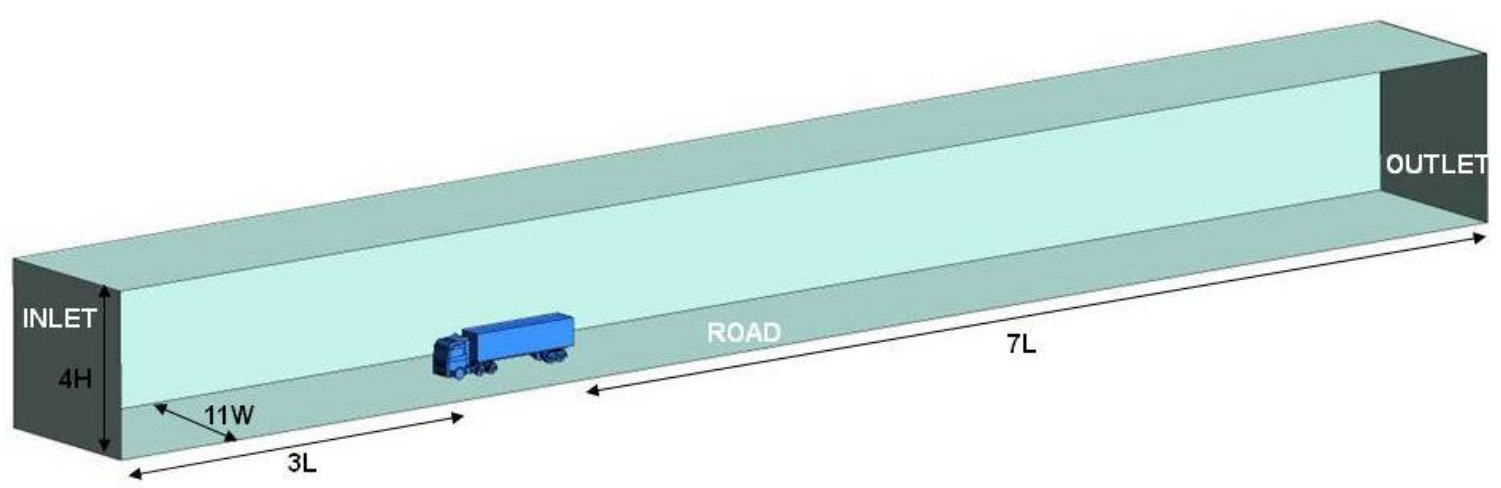

Figure 4. Numerical simulation of the calculation domain.

As shown in Figure 5, after some tentative studies, the tetrahedral meshes were used to create an unstructured grid system in the whole calculation domain, and the triangular surface meshes were used to better fit the shape of the model body. To catch the boundary layer from the model surface, there were six prism layers in the boundary, and the thickness was $0.4 \mathrm{~mm}$. Three layer density boxes were set up outside the baseline mode body, of which the minimum size was $8 \mathrm{~mm}$; there were more 
than ten million meshes for the case. The mesh and physics parameters used in ICEM software have been summarized in Table 2.

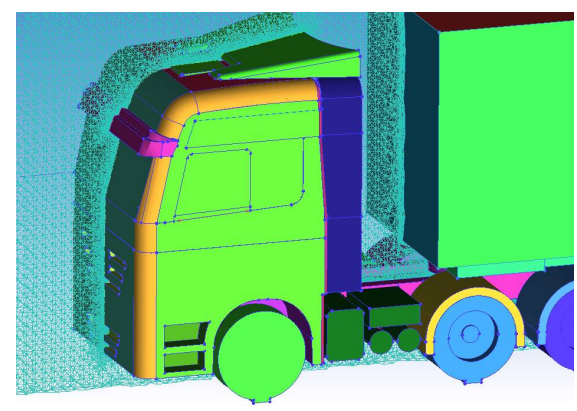

(a)

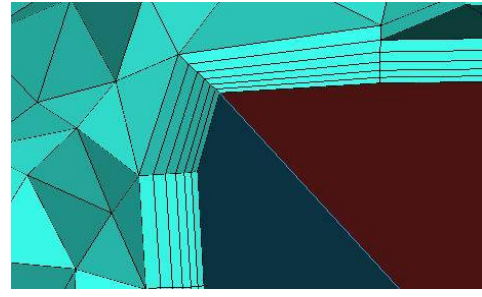

(b)

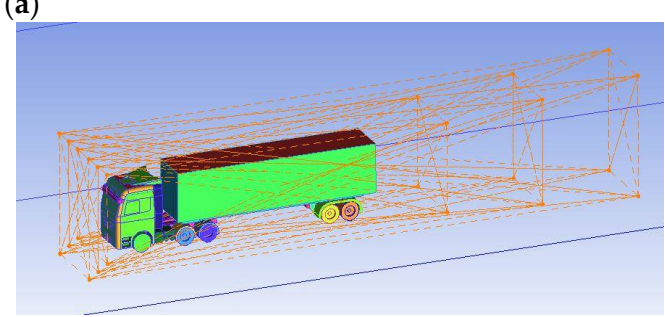

(c)

Figure 5. Numerical computation meshes: (a) surface mesh; (b) body surface boundary layer grid, and (c) density box of body. Each color shown different position of the cab body in ICEM software.

Table 2. Mesh and physics parameters.

\begin{tabular}{cc}
\hline Mesh Property & Parameter \\
\hline Surface mesh & Triangular mesh \\
Volume mesh & Tetrahedral mesh \\
Boundary layers & Prisms \\
Number of prism layers & 6 \\
Prism layers stretching & 1.1 \\
Prism layers thickness & $0.4 \mathrm{~mm}$ \\
Minimum cell size & $1 \mathrm{~mm}$ \\
\hline Physics Properties & Parameter \\
\hline Flow characteristics & Steady, stationary, incompressible, \\
Turbulence model & Shear Stress Transport k-omega \\
Flow velocity & $25 \mathrm{~m} / \mathrm{s}$ \\
\hline
\end{tabular}

The numerical simulation experiment was carried out by FLUENT (16.0, ANSYS Company, Canonsburg, PA, USA, 2015), which is based on the finite volume method. A steady and free-stream velocity of $U=25 \mathrm{~m} / \mathrm{s}$ was set at the inlet; the Reynolds number based on the hydraulic diameter was $1.95 \times 10^{6}$, and the turbulence intensity was $2.6 \%$. Through some tentative studies and consideration of the characteristics of the numerical simulation of vehicle external flow field [33-35], the Shear Stress Transport k-omega (SST k- $\omega$ ) turbulence model was adopted in this paper; meanwhile, convection term was solved by second-order upwind scheme, and coupling numeration of velocity field and stress was based on SIMPLEC scheme. The boundary condition of the outlet was pressure-outlet; no-slip condition was used on the lateral walls, roof, and the tractor-trailer baseline model surface.

\subsection{Validation of the Computational Model and CFD Procedure}

The purpose of the wind tunnel test carried out in this paper was to validate the correctness of the numerical simulation. Experiments were conducted in a closed-type reflux low-speed wind 
tunnel at Shenyang Aerospace University, and the test section had the dimensions of $3500 \mathrm{~mm}$ (length) $\times 1200 \mathrm{~mm}$ (width) $\times 1000 \mathrm{~mm}$ (height); the wind speed was varied in the range of $10-50 \mathrm{~m} / \mathrm{s}$, the contraction rate was $8: 1$, and the turbulence intensity of the freestream flow was less than $0.14 \%$. For the wind tunnel test, the cab of the tractor-trailer baseline model was made of wood, and the trailer and the remainder of the model were made of aluminum. The wheels were bolted to the model, and the cab roof fairing and both side deflectors were also constructed and bonded to the cab. In this paper, the free stream velocity was fixed at $U=25 \mathrm{~m} / \mathrm{s}$. The vehicle models installed in the test section had an insignifigant effect on the pressure distribution and the drag coefficient. The details of this wind tunnel test are shown in Figure 6.

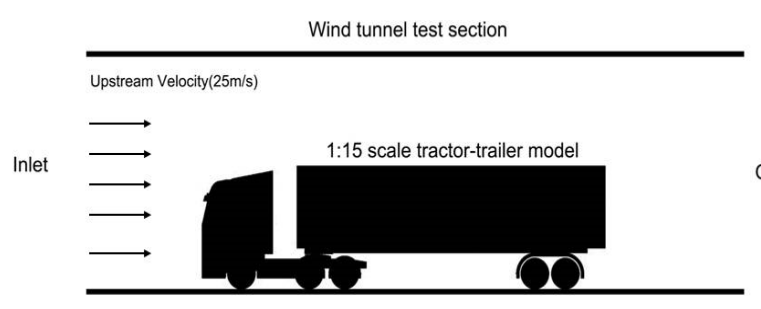

(a)

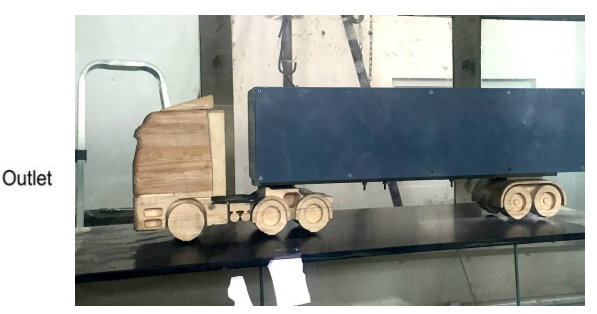

(b)

Figure 6. (a) Schematic diagram of the wind tunnel section and (b) photograph of the tractor-trailer.

The aerodynamic forces and moments of the tractor-trailer baseline test model were measured using a six-component force balance system. The drag force in the direction of the wind was determined by statistically averaging the data obtained from three independent measurements. The drag coefficient was calculated using the following equation:

$$
C_{d}=2 \cdot \frac{F}{\rho \cdot A \cdot V^{2}}
$$

in which $F$ is the drag force measured in the flow direction, $\rho$ is the fluid density, $V$ is the wind speed, and $A$ is the orthographic projection area of the tractor-trailer model. As a first step towards an improved approach, only a zero-yaw angle was tested in this study. In subsequent research, the aerodynamic drag coefficient $\left(C_{d}\right)$ of the baseline model was needed to compare the value of different parametric cab design tractor-trailer models to make the expression more explicit; the aerodynamic drag coefficient of baseline model was set as $C_{d \text {-baseline }}$.

During the numerical simulation of the baseline model, the aerodynamic drag coefficient was found to be stable after 1000 steps. The difference of the average value between 2000 and 2500 steps and 3501 and 4000 step was in the order of $10^{-4}$. Thus, in order to reduce the amount of calculation, the baseline model scheme was iterated by 3000 steps, and the average value of last 500 steps result was taken as the simulation value.

The $C_{d \text {-baseline }}$ values obtained by wind tunnel test and numerical simulation were 0.4507 and 0.4613 , respectively, as shown in Table 3. The relative error of the $C_{d}$ was $2.36 \%$; this estimation of the numerical number obtained from the simulation agreed well with the result measured in the wind tunnel experiments. This validated the grid division, the boundary conditions, and the physical model adopted for the simulation of the baseline model in this paper correctly, and has provided strong evidence for the validity and accuracy of the results obtained from the simulation.

For the numerical simulation of subsequent parametric cab design tractor-trailer models, only the length of calculation domain and the cab have changed; the CFD simulations of these cases still adopted the same mesh generation, iterated steps, and the numerical calculation scheme as the baseline model. 
Table 3. Drag coefficient of the tractor-trailer baseline model.

\begin{tabular}{cc}
\hline Experiment $\mathbf{V}=\mathbf{2 5} \mathbf{~} / \mathbf{s}$ & $C_{d \text {-baseline }}$ \\
\hline Wind tunnel & 0.4507 \\
Numerical simulation & 0.4613 \\
\hline
\end{tabular}

\section{Results and Discussion}

The $C_{d}$ is regarded as a major indicator that represents the aerodynamic performance of a vehicle; Table 4 illustrates the results of the CFD simulations of parametric designed tractor-trailer models with different cab variants. The impact of different geometrical modifications on the drag characteristics is shown in Table 4. In this table, $\Delta \boldsymbol{C}_{\boldsymbol{d}}$ represents the change rate of the $\boldsymbol{C}_{\boldsymbol{d}}$; its calculated equation is $\Delta \boldsymbol{C}_{\boldsymbol{d}}$ $=C_{d}-C_{d \text {-baseline }} / C_{d \text {-baseline, }}$ the symbol ' -' in the table represents the decreased of $C_{d}$; conversely, the symbol ' + ' represents the increased of $C_{d}$. It can be seen that in all cases, the $C_{d}$ decreased, except for the $400 \mathrm{~mm}-135$ degrees case, and the maximum drag reduction rate compared with the baseline model was about $8.49 \%$. These results imply that the protruding part of the cab and changing its dimensions have a distinct influence on the aerodynamic characteristics of tractor-trailers.

Table 4. Aerodynamic drag coefficient of new cab design models.

\begin{tabular}{|c|c|c|}
\hline Cab Model & $C_{d}$ & $\Delta C_{d}$ \\
\hline $400 \mathrm{~mm}-120$ degrees & 0.4298 & $-7.11 \%$ \\
\hline $400 \mathrm{~mm}-125$ degrees & 0.4351 & $-5.96 \%$ \\
\hline $400 \mathrm{~mm}-130$ degrees & 0.4341 & $-6.18 \%$ \\
\hline $400 \mathrm{~mm}-135$ degrees & 0.4982 & $+7.67 \%$ \\
\hline $500 \mathrm{~mm}-120$ degrees & 0.4402 & $-4.86 \%$ \\
\hline $500 \mathrm{~mm}-125$ degrees & 0.4234 & $-8.49 \%$ \\
\hline $500 \mathrm{~mm}-130$ degrees & 0.4281 & $-7.47 \%$ \\
\hline $500 \mathrm{~mm}-135$ degrees & 0.4260 & $-7.93 \%$ \\
\hline $600 \mathrm{~mm}-120$ degrees & 0.4459 & $-3.63 \%$ \\
\hline $600 \mathrm{~mm}-125$ degrees & 0.4501 & $-2.72 \%$ \\
\hline $600 \mathrm{~mm}-130$ degrees & 0.4280 & $-7.50 \%$ \\
\hline $600 \mathrm{~mm}-135$ degrees & 0.4442 & $-4.00 \%$ \\
\hline
\end{tabular}

It is interesting to note that when the angle between the protruding part and A pillar was fixed, the length of this part increased from $400 \mathrm{~mm}$ to $600 \mathrm{~mm}$; the $C_{d}$ first decreased and then increased. When the length was fixed in the $500 \mathrm{~mm}$ cases, the aerodynamic drag reduction was most pronounced. When the included angle was 120 degrees and the length of the protruding part was $400 \mathrm{~mm}$, the minimum $C_{d}$ was 0.4298 . When the included angles were 125 degrees, 130 degrees, and 135 degrees, the different parametric cab design vehicles with the $C_{d}$ were $0.4234,0.4281$, and 0.4260 , respectively, and the prominent length was $500 \mathrm{~mm}$; the $C_{d}$ was the smallest. It showed that when the extended length was $500 \mathrm{~mm}$, the rate of aerodynamic drag reduction was generally higher, and this dimension was a more suitable drag reduction length for the reference tractor-trailer model. However, when the protruding length was fixed, the change in $C_{d}$ was more complicated. When the extended length was $400 \mathrm{~mm}$ and $600 \mathrm{~mm}$, the aerodynamic $C_{d}$ first increased and then decreased with the increased of the angle. When the length was $500 \mathrm{~mm}$, the change of angle had little effect on aerodynamic drag.

The aerodynamic drag force on a vehicle is dependent on the form, and is caused by the pressure difference induced by massive flow separation. The present resolution was thought to be reasonable to investigate flow phenomena over the tractor-trailer and to explain the drag reduction mechanism. The 500 mm-125 degrees case had the most pronounced drag reduction effect; therefore, the numerical simulation results of this model were compared with the baseline model, and the related drag reduction mechanism was analyzed in Section 3.2. Meanwhile, due to the influence of the included angle and 
the length of protruding part, the change of $C_{d}$ was nonlinear; the influence of design variables is discussed in Section 3.3.

\subsection{Surface Pressure}

The air resistance of a driven vehicle is mainly determined by the forward positive pressure and the rear negative pressure. To investigate why the drag reduction mechanism was induced by the new design of the cab shape, the pressure distribution of the baseline model and the parametric cab design tractor-trailer needed to be analyzed.

Figure 7 displays the pressure contour comparison of the forebody of the cab between the baseline model and the 500 mm-125 degrees new design cab. As shown in Figure 7a, in the baseline model, a stagnant region can be observed on the front surface of the cab; there was a large area of positive pressure, and the front of visor and both sides of fairing also had positive pressure zones, which were the main sources of the aerodynamic resistance. The transition region between the 'front face' and the lateral part of cab showed negative pressure zones, indicating that there was a clear separation of the airflow. Both the positive and negative pressure zones extended from the bottom of the wheelhouse to the top of the front face. After modification of the design, as shown in Figure 7b, the upper surface of the protruding part of cab had a positive pressure area, compared with the baseline model; the positive pressure area of the front windscreen was smaller, and the size and area of the positive pressure at the fairing and visor were all reduced. At the same time, for the parametric design cab, the negative pressure zone of the transition position between the front windscreen and the side window had become smaller, but the value slightly increased, and the value of the negative pressure zone between the engine and side girth was reduced.
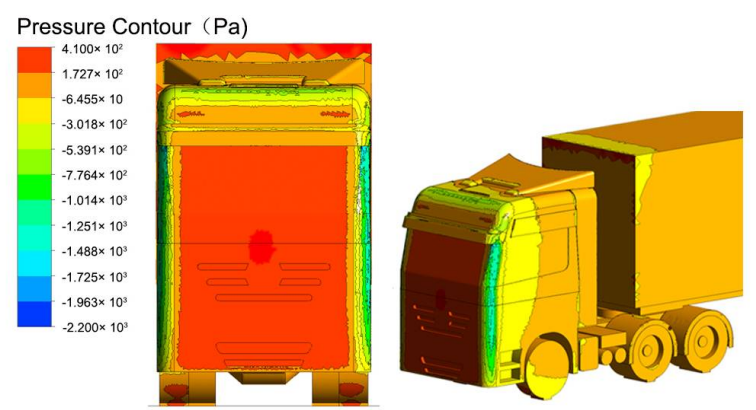

(a)
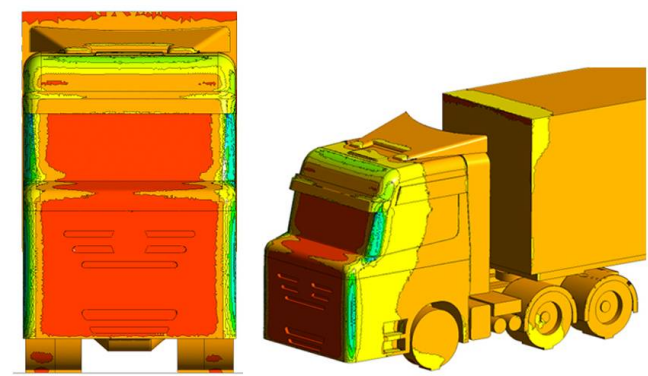

(b)

Figure 7. Pressure contour comparison of the front surface of the cab between (a) baseline model and (b) $500 \mathrm{~mm}-125$ degrees parametric cab design of the tractor-trailer.

In the baseline model as shown in Figure 8a, the pressure distribution of the front area of trailer was mainly a positive pressure zone. The obviously high pressure area that existed at the leading edge of the top and the front of the trailer, and the transition region between the front and both sides of trailer, were both striped. The shape of the cab influenced the flow conditions in front of the trailer; when the shape of cab was changed, the subsequent turbulence had changed too, and the 
pressure distribution of the front facade of the trailer was also affected. After the parametric cab design modification, as shown in Figure $8 \mathrm{~b}$, the positive pressure area of the trailer was reduced, where the high-pressure region at the front edge and both sides of the transition areas all became a thin sliver region. At the same time, the negative pressure zone at the front surface of the trailer model was significantly increased, whilst its location rose and was closed to the front edge of the trailer.

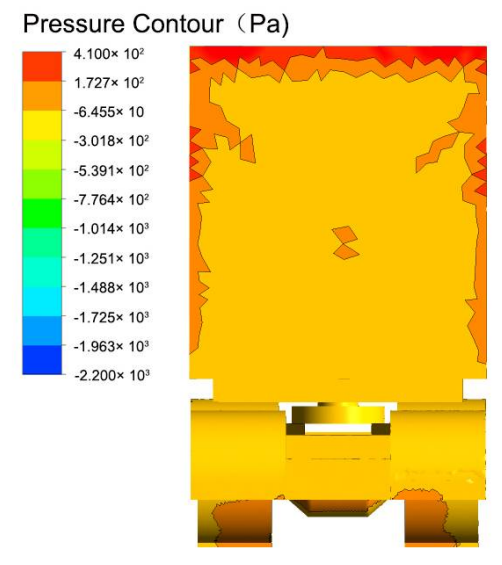

(a)

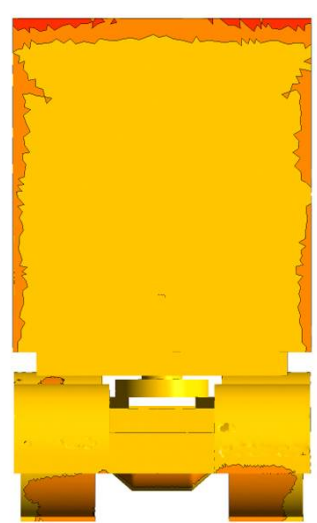

(b)

Figure 8. Pressure distribution comparison of the leading position of trailer between (a) baseline model and (b) $500 \mathrm{~mm}-125$ degrees parametric cab design of the tractor-trailer.

Figure 9a shows the pressure value comparison at the forebody between the baseline cab and the parametric design cab along the longitudinal symmetry plane. It can be seen from the line frame area (for the parametric design cab) at the position that near the protruding part, the positive pressure value was less than the baseline model; meanwhile, at the position above the protruding part, such as forward windshield and visor, the surface pressure has also been reduced too. Figure $9 \mathrm{~b}$ shows the pressure comparison at the forebody of the trailer between different cabs; it was also seen from the line frame area that after redesign, the positive pressure value decreased and the negative pressure value increased. From the comparison of the trend of the curves, the phenomenon of negative pressure area that had arisen was obvious, which corresponds to the display of the pressure contour (Figure 8).

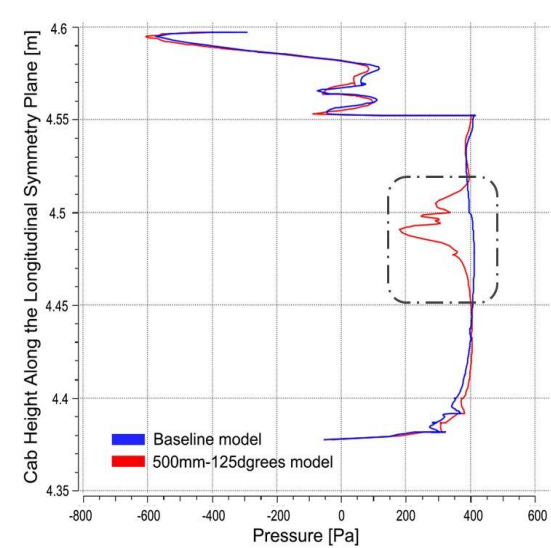

(a)

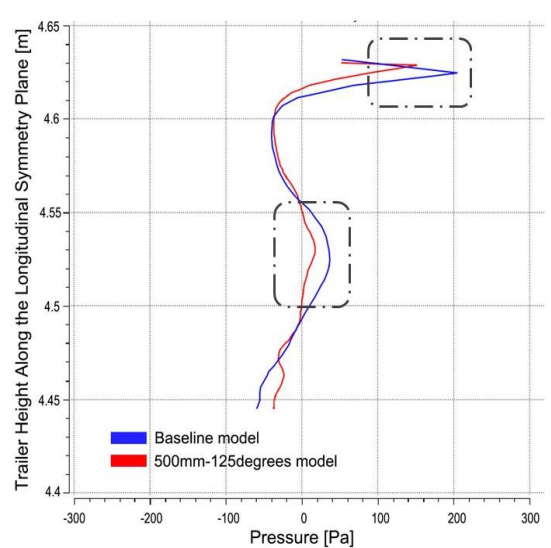

(b)

Figure 9. Cont. 


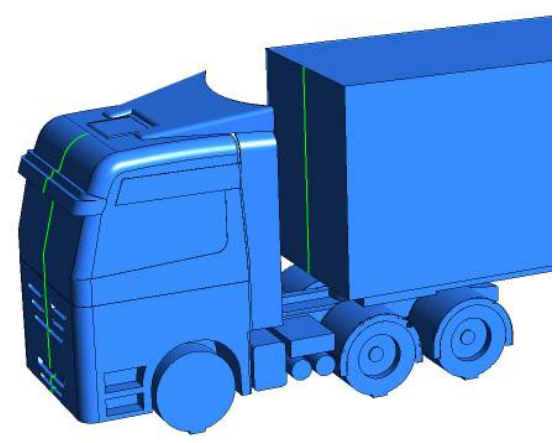

(c)

Figure 9. Pressure distribution contrast of tractor-trailers with different cab design along longitudinal symmetry plane between the (a) the fore body of the cab and (b) the forebody of the tailer; (c) pressure measurement position with baseline model as an example. The vehicle model was displayed in blue, longitudinal symmetry line along the driving direction was displayed in green.

\subsection{Change of Flow Field}

The time-averaged flow fields around the parametric designed cab tractor-trailer models have been presented in this section. When the incoming flow reached the front of the baseline cab model, part of the flow was halted by the front face of the model. As a result, the flow stagnation region appears on the front surface of the baseline cab model. The flow above the stagnation point turned upwards and reached the top of the cab, and after turning upwards, the flow reached and expanded along the surface of the fairing and the roof of the cab. The flow then reached the rear end of the cab, and some of the flow entered the gap between the cab and the trailer; at the same time, the rest of the incoming flow crossed the gap and impinged on the front surface of the baseline trailer model.

From Figure 10, it can be seen after the parametric designed case was applied (500 mm-125 degrees) that when the incoming flow reached the new design of the cab, the flow attacked the 'cab nose' first and immediately halted. Due to the friction of the 'cab nose' surface and the blocking effect of the front windscreen, a vortex formed here that rotated clockwise at the top of the 'cab nose'. Consequently, under the lift action from this vortex, the incoming flow above it changed direction and turned upward to the top of the cab's front face and the visor. Some of the airflow even went over the visor with the assistance of this vortex and directly reached the front edge of the cab. At the same time, because of the vortex, the upward incoming flow contacted the cab and developed relative motion instead of making surface contact with the cab body. Compared to the friction between the airflow and the cab surface, the friction between the airflows apparently decreased. Therefore, the vortex that existed above the 'cab nose' can be defined as it has a 'vortex cushion' effect; the actual function of this vortex was even like a diversion device. It not only effectively reduced the frictional resistance of the airflow, but it also acted as a lift to speed up the airflow. It was believed that the presence of the 'vortex cushion' on the 'cab nose' induced changes in the incoming flow filed, thus varying the pressure distribution and the aerodynamic drag force, corresponding to Figure $6 \mathrm{~b}$ and Table 4 , as shown.

The structure of the flow that passed over the front face of the cab was changed obviously, and its subsequent flow was bound to be affected. As can be seen in Figure 11b, compared to the baseline model (Figure 11a), the position of the upper vortex core that was located in the gap region between the new design of the cab and trailer obviously lifted and moved closer to the rear edge of the fairing, whilst the horizontal position of this vortex was close to the front edge of the trailer. This phenomenon was the main cause of the elevation of the negative pressure zone, which was in front of the surface of the trailer, as shown in Figure 8b. Because of this upper vortex lift, the location of the upper backflow stagnated on the rear surface of the new design of the cab and was lifted accordingly; meanwhile, this left more space for the motion of the bottom vortex at the gap, and the shape of this bottom vortex was more complete. The variety in the flow structure at the gap corresponded to the change of the 
pressure distribution on the front surface of the trailer and resulted in the positive pressure decreasing and the negative pressure increasing; the aerodynamic pressure resistance of the whole tractor-trailer was reduced.

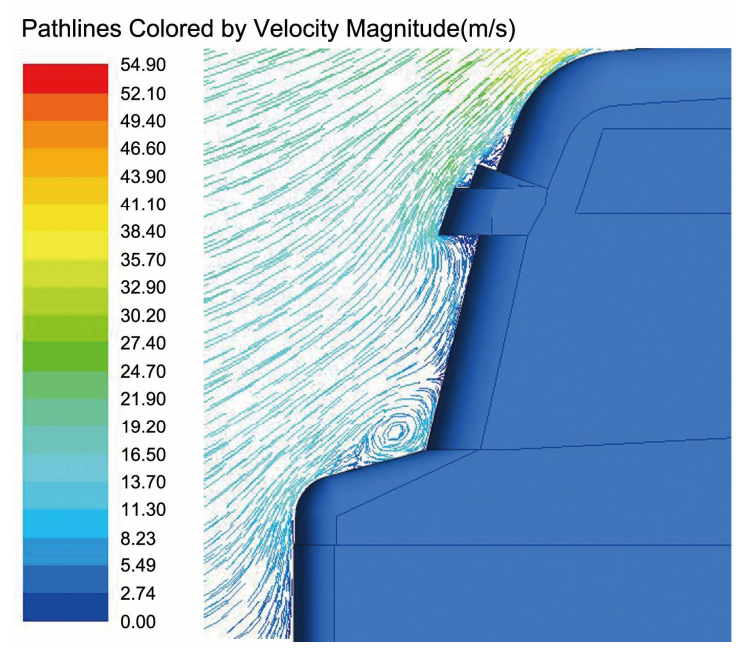

Figure 10. Flow field structure of protruding part of $500 \mathrm{~mm}-125$ degrees parametric cab design of the tractor-trailer.

Pathlines Colored by Velocity Magnitude(m/s)

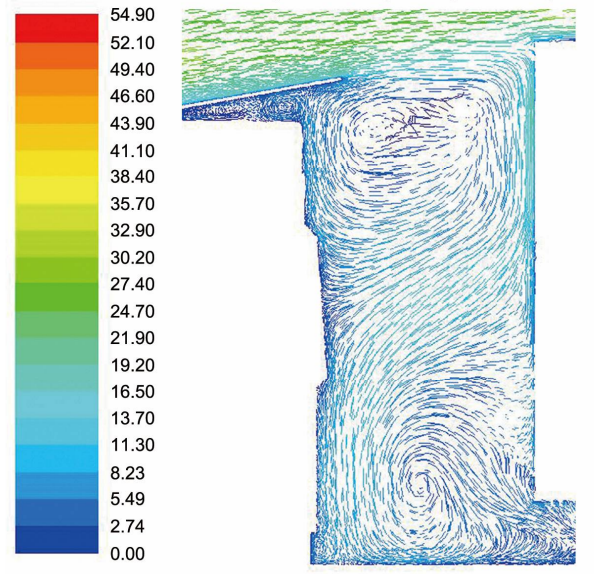

(a)

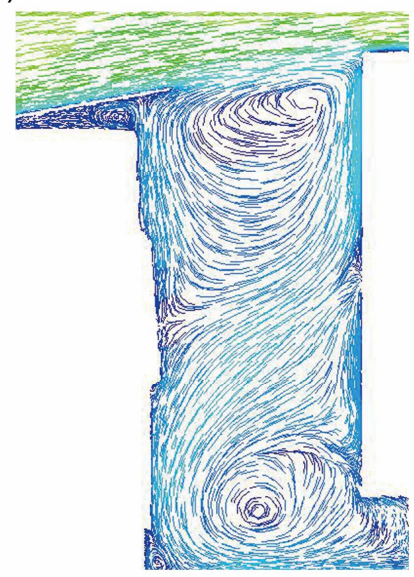

(b)

Figure 11. Flow field comparison of the gap between (a) baseline model and (b) $500 \mathrm{~mm}-125$ degrees parametric cab design of the tractor-trailer.

Figure 12 shows the contrast of the longitudinally symmetric turbulent cloud diagram between the baseline model and the new design cab model; the results obtained earlier (Figures 10 and 11) are also reflected in this picture. The flow separation appeared at the bottom of the original cab and the turbulence intensity was also larger there. At the leading edge of the fairing, the flow was blocked after it impinged on the mounting base of the fairing, but most parts of the flow still moved along the curved surface of the fairing to the rear until they collided with the front of the trailer. 
Turbulent Intensity Contours (\%)

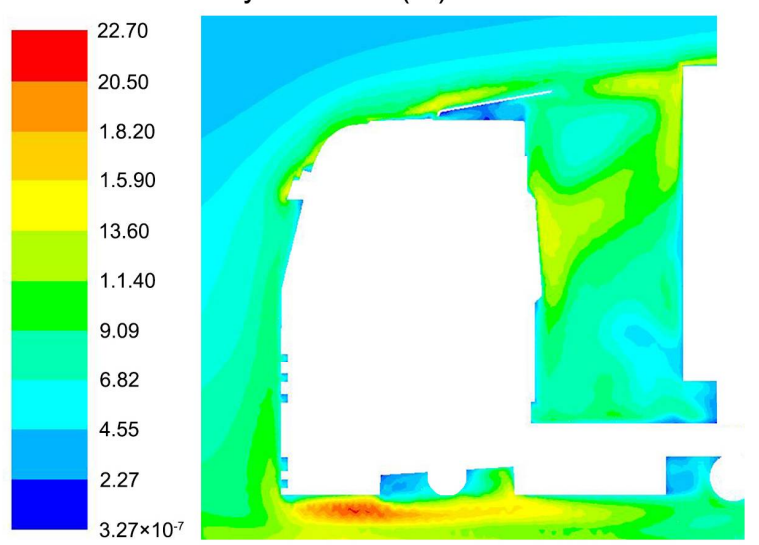

(a)

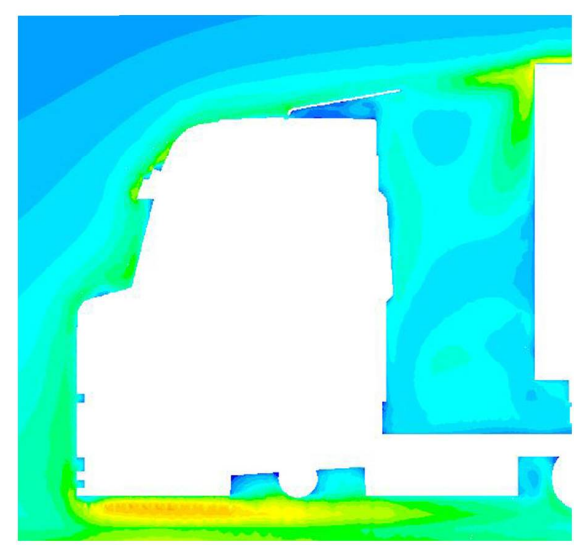

(b)

Figure 12. Turbulence intensity comparison of (a) baseline model and (b) $500 \mathrm{~mm}-125$ degrees parametric cab design of tractor-trailer.

The airflow separation at the bottom of the new design of the cab changed greatly, and the turbulence intensity at this point was obviously smaller than that of the original model. This means that after the parametric cab design, the air flow was smoother, and the shunting action of the cab's protruding 'cab nose' began to be highlighted. At the same time, the obvious change of the turbulence intensity at the gap between the cab and trailer was also caused by the 'vortex cushion' effect of the vortex above the protruding 'cab nose'. From functional viewpoint, this vortex acted as a 'vortex cushion'; it even could be seen as a part of the cab, and combined with its shape, it also could be considered that the cab has formed a certain streamline design here. The existence of the 'vortex cushion' effect made the flow over the front and top of the cab smoother, and the diversion effect of the fairing on the cab was more pronounced.

Figure 13 shows the wake structure comparison between the baseline model and the parametric cab design tractor-trailer. As shown in Figure 13b, after the cab redesigned, there were also two vortices that occurred at the rear of the trailer; one was higher up and the other one was lower down. Compared to the baseline model, in the wake flow of the $500 \mathrm{~mm}-125$ degrees model, the position of the upper vortex was obviously closer to the rear of the vehicle and had moved upwards, and its vortex area was smaller. Accordingly, the form of the lower vortex in the wake flow was more complete, its height was reduced, and the core of the lower vortex was closer to the vehicle too. From comparison of the wake structure of the different tractor-trailers after the new cab was designed, the separation area of the wake flow showed a decreasing trend, and its location was concentrated more at the rear of the trailer.

Figure 14 shows the turbulent intensity contour comparison of wake flow of different tractor-trailer models. The parametric design of cab also had a certain influence on the tail flow structure. Comparing the different tractor-trailers, it was interesting to note that the turbulence intensity of the parametric cab design vehicle was obviously smaller than that of the baseline model, as shown in Figure 14 . The 'vortex cushion' of the 'cab nose' not only impacted the flow structure of the cab and the gap but also influenced the vortex structure of the boundary layer at the bottom of the trailer, and this meant that the dynamic exchange of the upper and lower layers of the boundary layer were weakened and the momentum transport was smoother; finally, the aerodynamic drag force of the tractor-trailer was decreased. 


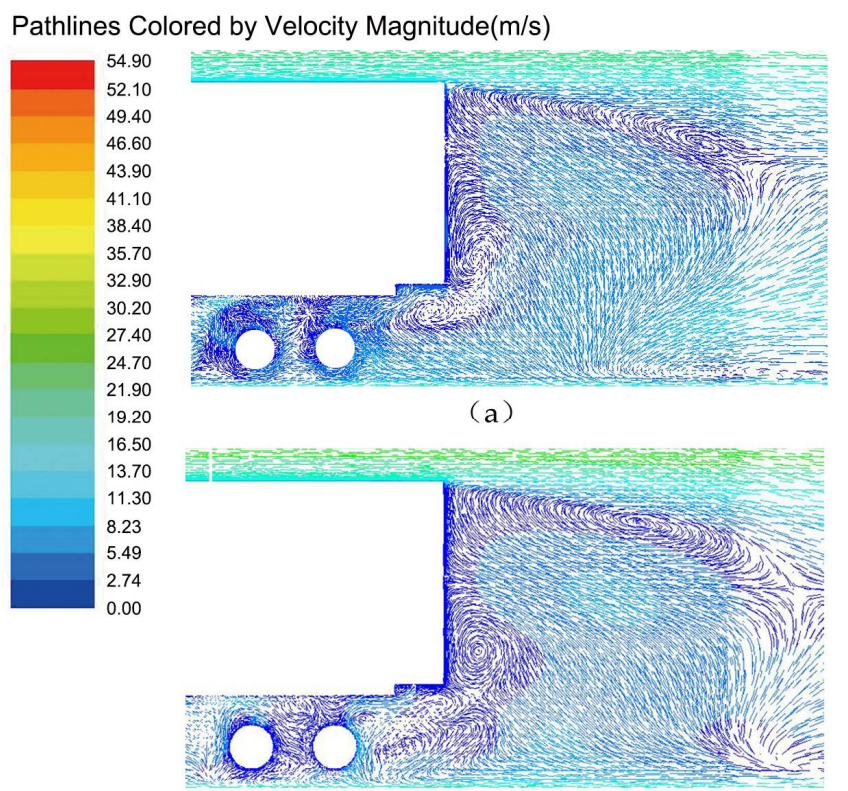

(b)

Figure 13. Flow field comparison of (a) baseline model and (b) $500 \mathrm{~mm}-125$ degrees parametric cab design of the tractor-trailer.

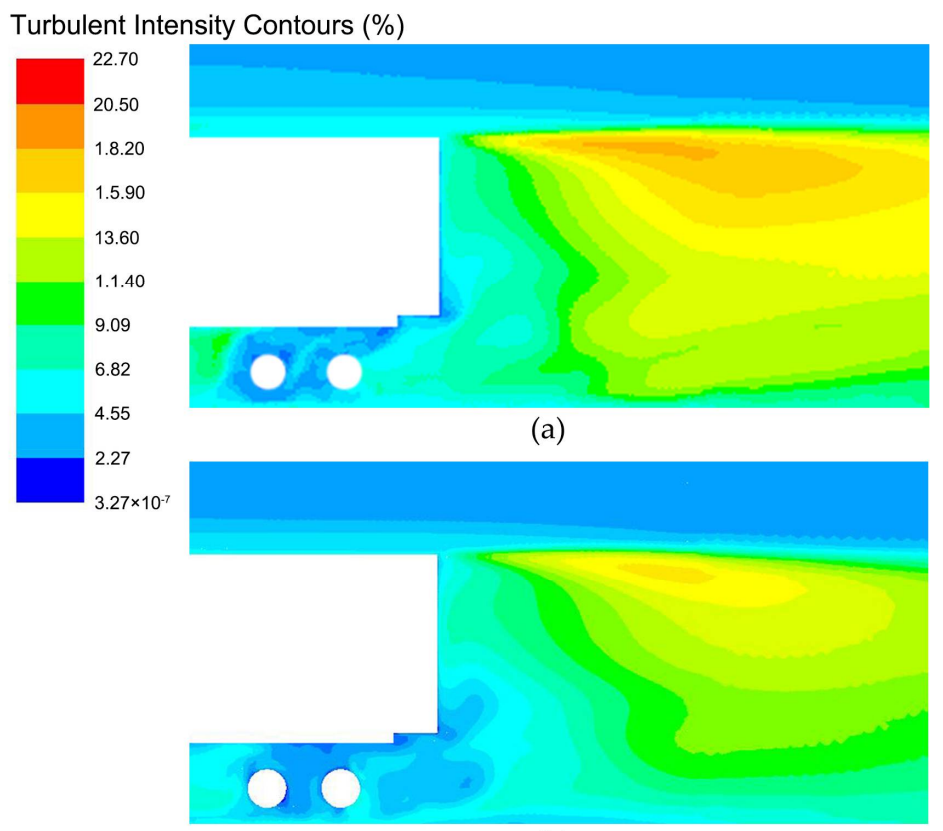

(b)

Figure 14. Turbulence intensity contour comparison of rear part: (a) baseline model and (b) $500 \mathrm{~mm}-125$ degrees parametric cab design of the tractor-trailer.

\subsection{Impact Factors of the 'Vortex Cushion'}

In Section 3.2, it was found that a vortex formed above the protruding part and obviously influenced the pressure distribution and the flow field of the tractor-trailer. As shown in Table 4, when the angle between the protruding part and the A pillar was greater than 125 degrees, with the protruding part length increased, the $C_{\boldsymbol{d}}$ decreased firstly then increased subsequently. Therefore, the formation of this 'useful vortex' depended on the friction of the protruding part surface and the 
blocking effect of the front windscreen, and must have been affected by the two design variables: the length of the protruding part and the angle between this part and the A column.

As shown in Figure 15a, when the extended length was $400 \mathrm{~mm}$, it was slightly protruded, and the above vortex area was obviously smaller than other two cases. At the position of the include angle between protruding part and A pillar, the flow velocity was smallest. Owing to the small vortex volume, a part of the incoming flow contacted the vortex above the 'cab nose' first and then continued to move forward and impinge on the front windshield. The other part of the flow went down to the sun shading board, and the movement was blocked. At this time, the effect of 'vortex cushion' was not obvious.

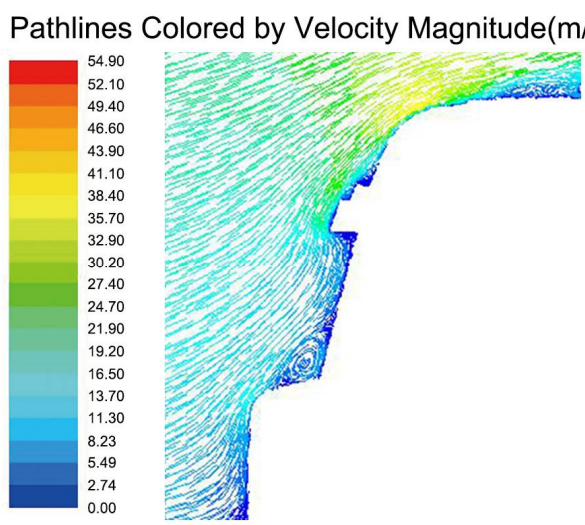

(a)

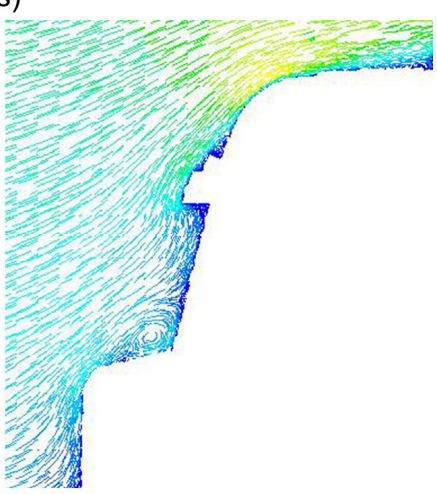

(b)

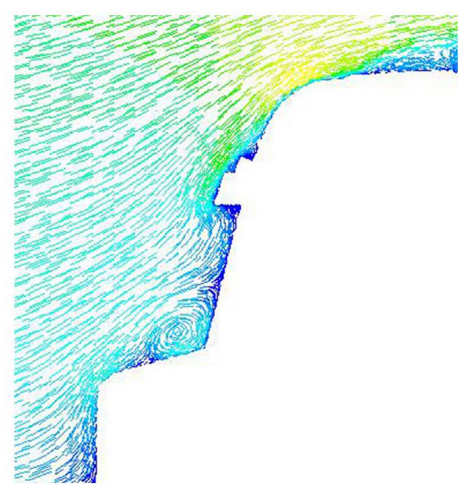

(c)

Figure 15. Flow field of different new designs of the cab: (a) $400 \mathrm{~mm}-125$ degrees, (b) $500 \mathrm{~mm}-125$ degrees, and (c) $600 \mathrm{~mm}-125$ degrees parametric cab design of the tractor-trailer.

When the extended length was $500 \mathrm{~mm}$, as shown in Figure $5 \mathrm{~b}$, the vortex was fully developed, its size was larger than $400 \mathrm{~mm}$ case, and the shape was even rounder and fuller. At this moment, at the include angle, the flow velocity was faster, and the bearing role of this vortex began to be highlighted. The friction between the airflows was apparently smaller than the friction between the airflow and the cab surface; therefore, compared with the $400 \mathrm{~mm}-125$ degrees case and the baseline model, the value of $C_{d}$ decreased.

When the extended length was $600 \mathrm{~mm}$, the vortex did not increase linearly, but also kept a size similar to the $500 \mathrm{~mm}$ case, and the effect of 'vortex cushion' was not enhanced too. There was still a certain length at the front corner of the protruding 'cab nose' that was not occupied by the vortex. The incoming air flow to the cab was first affected by the surface of the protruding position, and then it impinged on the vortex. Some of the airflow was blocked by the vortex, and the velocity was reduced; the other part continued to flow upward with the lifting of the vortex and at a later point the flow impinged on the cab's sunshade. Meanwhile, the friction between flow and vehicle surface was greater than $500 \mathrm{~mm}$ case, and the value of $\boldsymbol{C}_{\boldsymbol{d}}$ increased. Therefore, for such a forward protruding 'cab nose' type of cab shape design, the protruding length needs to refer to the overall size and proportional relationship of the cab.

The same results also could be obtained in Figure 16; as shown in Figure 16a, the area of gray dash wire frame was the surface of the protruding part, and the value of pressure of $500 \mathrm{~mm}$ case was less than $400 \mathrm{~mm}$ case clearly and close to the value of the $600 \mathrm{~mm}$ case. In the area of purple dot dash line, the surface pressure of $500 \mathrm{~mm}$ case was obviously less than other two cases; correspondingly, the value of $C_{d}$ decreased first and then increased as the length of protruding part increased. 


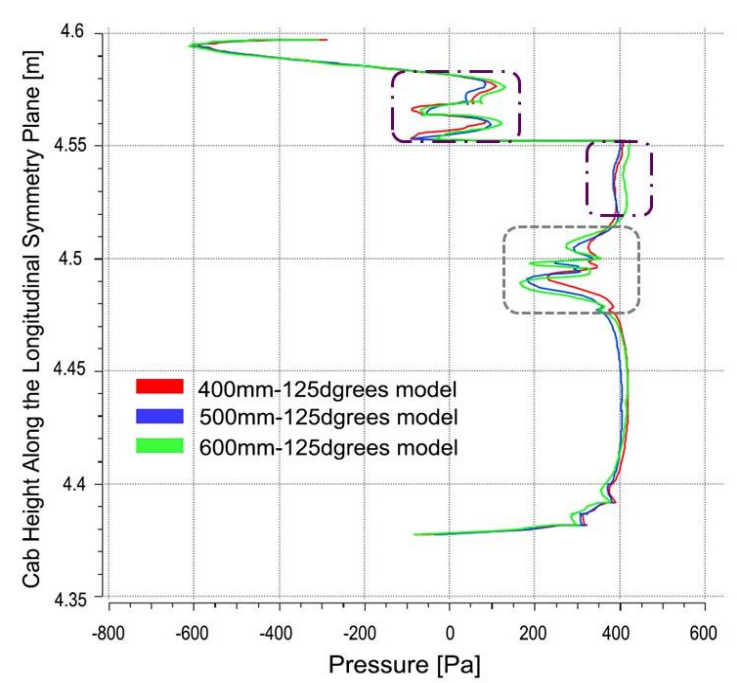

(a)

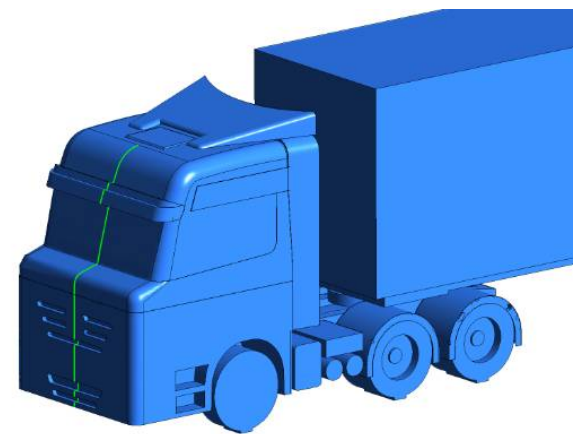

(b)

Figure 16. Pressure distribution contrast along longitudinal symmetry plane of tractor-trailer cabs with different parametric designs: (a) 125 degrees design schemes and (b) pressure measurement position with $400 \mathrm{~mm}-125$ degrees scheme as an example.

Figures 17 and 18 show the velocity and turbulent kinetic energy contour at the same position when the included angle was 125 degrees and with different extended lengths of the cab. When the length of the protrusion was smaller, this value relative to the whole size of the tractor was not obvious, and the cab could still be regarded as a blunt body without a 'car nose'; the separation of the airflow here was still similar to that of the flat head cab. However, when the protrusion length was increased, its proportion relative to the cab was raised too, and the effect on the separation of the airflow could not be ignored. The cab was somewhat similar to the design of the "wedge" car. It can be clearly seen from Figure 16 that with the increase of the protrusion length, the separation of the flow tended to be gentler at the angle that at between the protruding part and the front windshield, and the area of the separation region was gradually decreased. From Figure 17, it can be seen that the change of the turbulent kinetic energy at the lower part of the cab could also directly capture the characteristics of this 'wedge' design, similarly to the function of the car engine bay that inserts it into the flow and guides it to move to the rear.

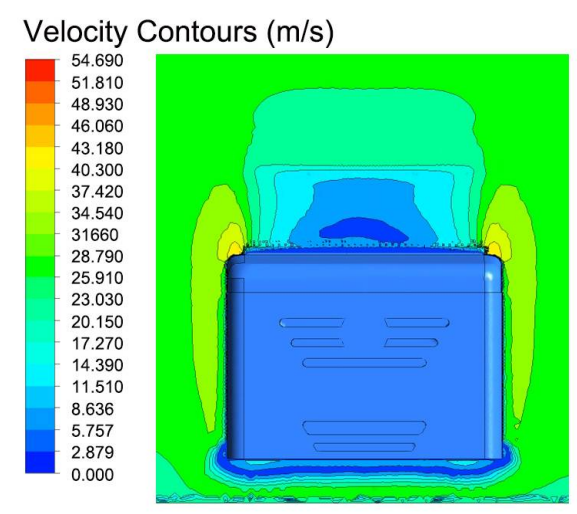

(a)

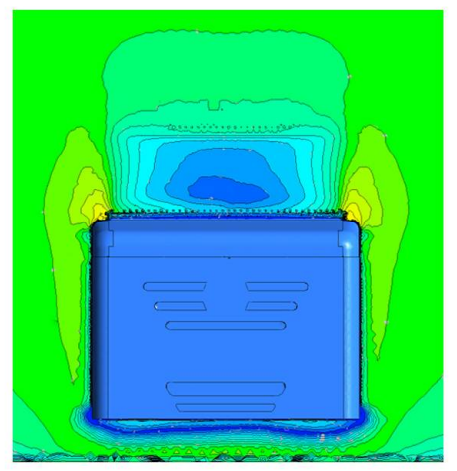

(b)

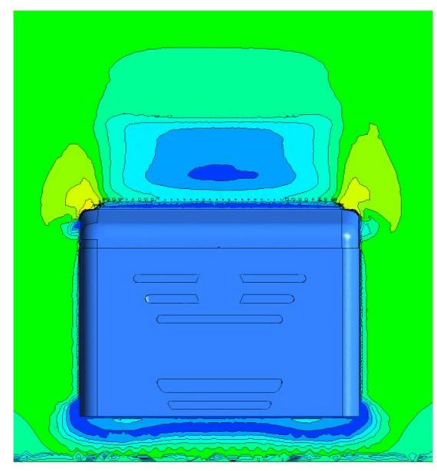

(c)

Figure 17. Comparison of the velocity contour at the angle between the protruding part and the front windshield between (a) 400 mm-125 degrees, (b) 500 mm-125 degrees, and (c) 600 mm-125 dgrees parametric cab design of the tractor-trailer. 


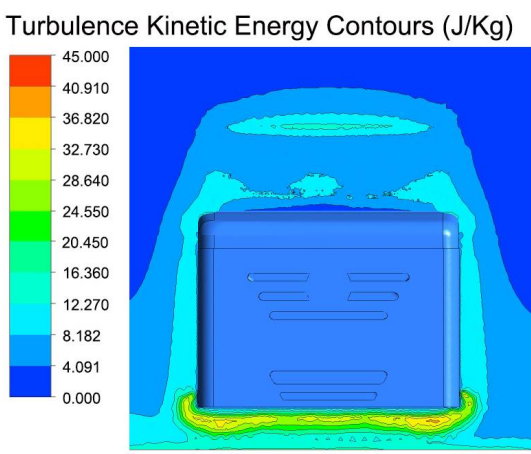

(a)

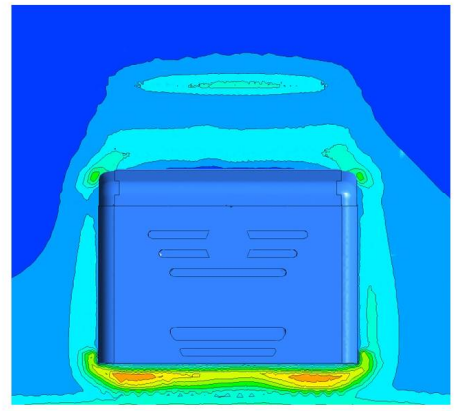

(b)

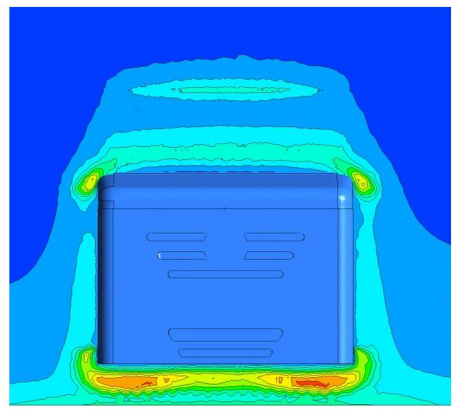

(c)

Figure 18. Comparison of the turbulent kinetic energy contour at the angle between the protruding part and the front windshield between (a) 400 mm-125 degrees, (b) 500 mm-125 degrees, and (c) 600 $\mathrm{mm}-125$ degrees parametric cab design of the tractor-trailer.

It was found from Table 4 that when the protruding part was $400 \mathrm{~mm}$ and the angle between the protruding part and the A pillar was 135 degrees, the $C_{d}$ of the tractor-trailer did not decrease as expected, but increased by $7.67 \%$. However, when the protruding length was $500 \mathrm{~mm}$ and $600 \mathrm{~mm}$, the aerodynamic drag was still reduced. Therefore, it was also necessary to analyze the cases when the angle was larger, as well as the relationship between the protruding part length and the outflow structure of the parametric cab design tractor-trailer.

Figure 19 shows the velocity pathlines diagram of the longitudinal symmetry plane of the cab when the angle was 135 degrees. It is interesting to note that the vortex that acted as a 'vortex cushion' disappeared when the length of protruding part was $400 \mathrm{~mm}$. At the same time, when the extended length was $500 \mathrm{~mm}$ and $600 \mathrm{~mm}$, compared with other two cases of the same length of 125 degrees and 130 degrees, the size of the vortex was obviously smaller. The appearance of this vortex not only depended on the blocked windshield by the front, but also on the friction between the airflow and the 'cab nose' surface. When the angle was too large, the frictional force was affected when the airflow travelled on a tilted slope, and meanwhile the blocking effect of the front windshield was weakened. When the design length was $400 \mathrm{~mm}$, this length was not enough to form the corresponding vortex, and at the same time, with the rounded corners of the leading edge of the protruding part, the incoming airflow could not discriminate the angle, instead turning it 'as a big blunt form' and continuing to flow upward. This is one of the main reasons for the significant increase of aerodynamic drag in the $400 \mathrm{~mm}-125$ degrees new cab design. Compared to the $500 \mathrm{~mm}$ and $600 \mathrm{~mm}$ cases, there was enough length to form a vortex, but the area of the vortex was significantly reduced. The 'vortex cushion' effect also existed, but the effect only to led the airflow flow to the upper part of the windshield, which had no fundamental influence on the outflow field structure.

The characteristics of the vortex and its 'vortex cushion' effect were also influenced by the angle between the protruding part and the A pillar. When the included angle was only 120 degrees, as shown in Figure 20a, the incoming flow was impacted by the friction from the protruding part and then blocked by the front windshield glass, leading to the vortex largest, and this vortex was also blocked and rubbed the above airflow. To a certain extent, in addition to completing the role of a certain 'vortex cushion', this vortex also obstructed the flow. When the protruding part was gradually tilted and the angle became larger, the area of the vortex decreased; as shown in Figure 20b,c, the function of the 'vortex cushion' began to be highlighted. But when the include angle was too large, as shown in Figure 20d, the frictional force derived from the protruding part was not enough to form a certain area of the vortex due to the influence of the angle, and the 'vortex cushion' effect did not exist either. Only when the angle was changed to a certain reasonable range could the vortex play an effective role in supporting the external air flow and reduce the aerodynamic drag. 
Pathlines Colored by Velocity Magnitude $(\mathrm{m} / \mathrm{s})$

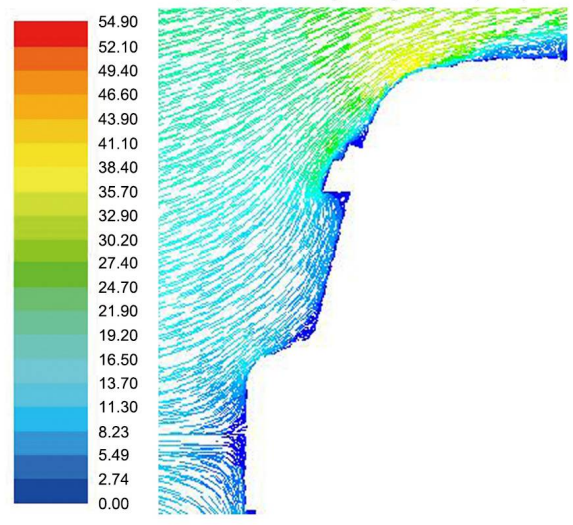

(a)

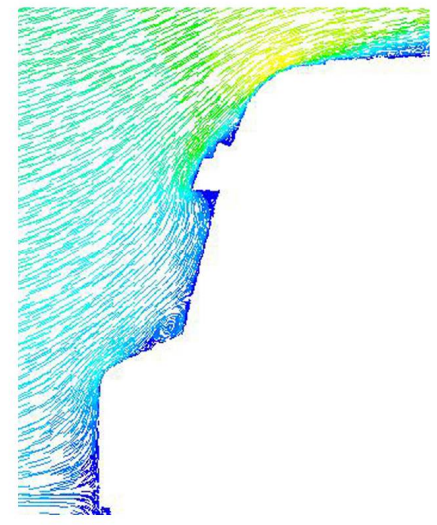

(b)

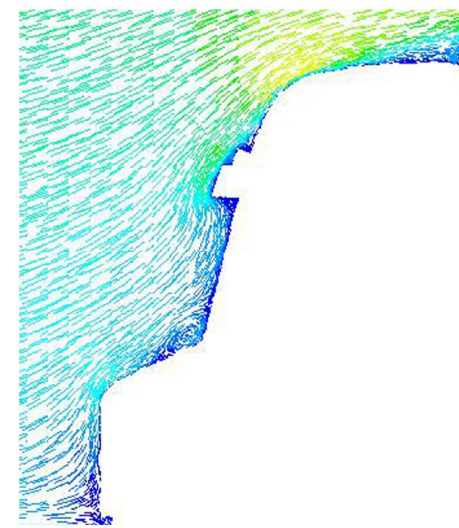

(c)

Figure 19. Longitudinally symmetric surface velocity pathlines with different lengths: (a) $400 \mathrm{~mm}$-135 degrees, (b) $500 \mathrm{~mm}$-135 degrees, and (c) $600 \mathrm{~mm}-135$ degrees parametric cab design of the tractor-trailer.

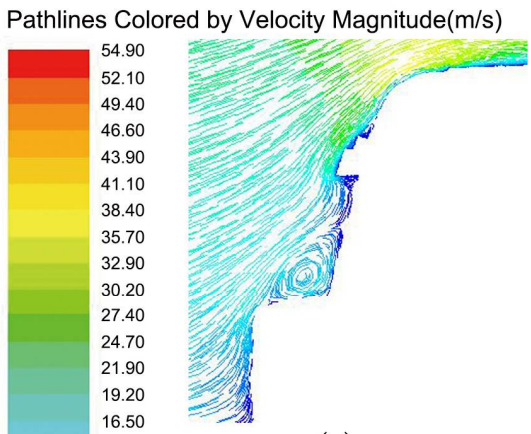

(a)

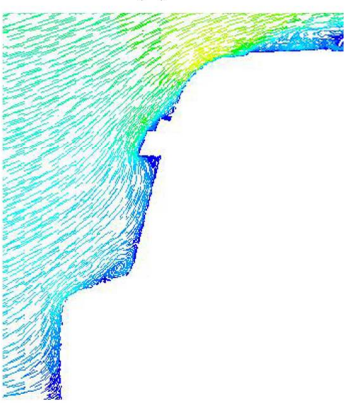

(c)

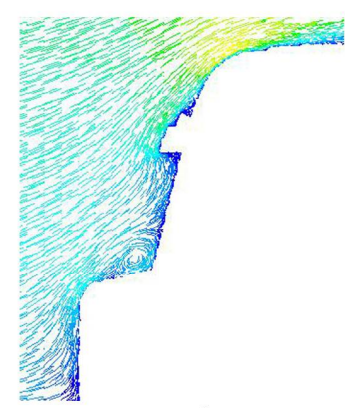

(b)

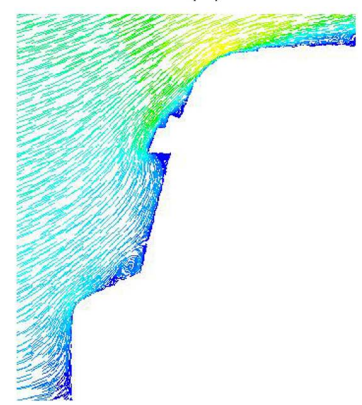

(d)

Figure 20. Longitudinally symmetric surface velocity pathlines with (a) $500 \mathrm{~mm}-120$ degrees, (b) 500 mm-125 degrees, (c) 500 mm-130 degrees, and (d) 500 mm-135 degrees parametric cab design of the tractor-trailer.

\section{Conclusions}

In this study, parametric designed cabs, which were inspired by the external forebody shape of the cheetah, have been proposed to investigate the possible effect of drag reduction on tractor-trailers. An actual articulated tractor-trailer commonly used in China was used as a baseline model, and its drag coefficient value was 0.4507 . The drag coefficients of twelve parametric cab design cases were quantitatively evaluated by CFD simulation. Among these experimental results, the optimum $500 \mathrm{~mm}-125$ degrees cab design largely reduced the drag coefficient of the tractor-trailer model by $8.49 \%$; essentially, a minimum drag coefficient was achieved in all cases, except in the case of 
the $400 \mathrm{~mm}-135$ degrees model. The flow field of the baseline model and the tractor-trailer with a parametric cab design model were analyzed, including the forebody of the cab, the gap between the $\mathrm{cab}$ and trailer, and the rear of the tractor-trailer, to understand the drag reduction mechanism. After the parametric cab design, because of the friction of the vehicle surface and the obstructed effect of the windshield, there was a vortex formed above the protruding part, and this vortex acted as a 'vortex cushion' to accelerate the speed of the flow; accordingly, the positive and negative pressure distribution of the front surface of the cab and trailer were changed, and therefore the aerodynamic drag decreased. Moreover, the influence of the length of the protruding part and the angle between it and the A pillar on the 'vortex cushion' have also been discussed in this paper; these two design variables affected the size of vortex and the friction between the air flow and vehicle surface, so the $C_{d}$ of the tractor-trailer was changed accordingly. The findings of this study have provided useful information for tractor-trailers to reduce aerodynamic drag and enhance fuel economy with the method of parametric cab design.

Author Contributions: All authors contributed to the research work. T.W. conceived and designed the wind tunnel and simulation experiments; J.P. and X.S. performed the experiments and analyzed the data; J.P. reviewed the research work and wrote the paper. T.Y. and G.L. participated in the experiments.

Funding: This research was funded by the High Technology Industrialization Project of Shanxi Province of China (Grant 2011-2368). Without its financial support, this work would not have been possible.

Acknowledgments: Thanks are due to Ruiliang Zhang, Huan Yin and Jingyuan Yao of Taiyuan University of Technology for valuable discussion, to Guowen Li and Chengjun Wang of Shenyang Aerospace University for their help in modelling and testing.

Conflicts of Interest: The authors declare no conflict of interest.

\section{References}

1. Hucho, W.-H.; Sovran, G. Aerodynamics of Road Vehicles. Annu. Rev. Fluid Mech. 1993, 25, 485-537. [CrossRef]

2. William, F. Milliken, Douglas, L. Milliken. Race Car Vehicle Dynamics; SAE International: Warrendale, PA, USA, 1995.

3. Patel, P.; Vijayakumar, S. External Flow Analysis over a Car to Study the Influence of Different Body Profiles Using CFD; SAE Technical Paper 2001-01-3085; Ministry of Higher Education/Baghdad University: Baghdad, Iraq, 2001.

4. Croll, R.H.; Gutierrez, W.T.; Hassan, B.; Suazo, J.E.; Riggins, A.J. Experimental Investigation of the Ground Transportation Systems (GTS) Project for Heavy Vehicle Grag Reduction. In Proceedings of the International Congress \& Exposition of the Society of Automotive Engineers, Detroit, MI, USA, 1 February 1995.

5. Gutierrez, W.T.; Hassan, B.; Croll, R.H.; Rutledge, W.H. Aerodynamics Overview of the Ground Transportation Systems (GTS) Project for Heavy Vehicle Drag Reduction; SAE Technical Paper 960906; Sandia National Labs: Albuquerque, NM, USA, 1996.

6. McCallen, R.; Browand, F.; Ross, J. The Aerodynamics of Heavy Vehicles: Trucks, Buses, and Trains, Berlin; Springer: New York, NY, USA, 2004.

7. Hyams, D.G.; Sreenivas, K.; Pankajakshan, R.; Nichols, D.S.; Briley, W.R.; Whitfield, D.L. Computational simulation of model and full scale Class 8 trucks with drag reduction devices. Comput. Fluids 2011, 41, 27-40. [CrossRef]

8. Östh, J.; Krajnović, S. The Flow around a Simplified Heavy Truck Model Studied by Large Eddy Simulation. J. Wind Eng. Ind. Aerodyn. 2011, 102, 36-47. [CrossRef]

9. Dasa, P.; Tsubokuraa, M.; Matsuuki, T.; Oshima, N.; Kitoh, K. Large Eddy Simulation of the Flow-field Around a Full-Scale Heavy-Duty Truck. Procedia Eng. 2013, 56, 521-530. [CrossRef]

10. Cooper, K.R. Truck Aerodynamics Reborn-Lessons from the Past; SAE Technical Paper 2003-01-3376; Aerodynamics Laboratory, NRC Canada: Ottawa, ON, Canada, 2013.

11. Krishna, M.V.; Ram, C.V. Adjustable Roof Fairing for Truck Aerodynamics; SAE Technical Paper 2011-26-0106; Ashok Leyland Technical Centre: Tamil Nadu, India, 2011.

12. Kim, J.J.; Lee, S.; Kim, M.; You, D.; Lee, S.J. Salient Drag Reduction of a Heavy Vehicle Using Modified Cab-Roof Fairings. J. Wind Eng. Ind. Aerodyn. 2017, 164, 138-151. [CrossRef]

13. Kim, J.J.; Kim, J.; Lee, S. Substantial Drag Reduction of a Heavy Truck Vehicle Using Gap Fairings. J. Wind Eng. Ind. Aerodyn. 2017, 171, 93-100. [CrossRef] 
14. Khosravi, M.; Mosaddeghi, F.; Oveisi, M.; Khodayari, B.A. Aerodynamic Drag Reduction of Heavy Vehicles Using Append Devices by CFD Analysis. J. Cent. South Univ. Technol. 2015, 22, 4645-4652. [CrossRef]

15. Hwang, B.G.; Lee, S.; Lee, E.J.; Kim, J.J.; Kim, M.; You, D.; Lee, S.J. Reduction of Drag in Heavy Vehicles with Two Different Types of Advanced Side Skirts. J. Wind Eng. Ind. Aerodyn. 2016, 155, 36-46. [CrossRef]

16. Hu, X.; Yang, B.; Guo, P.; Wang, J.-Y.; Yang, Y.; An, Y. Aerodynamic Drag Reduction of Van Based on Underbody Skirt. J. Jilin Univ. 2011, 41 (Suppl. S2), 108-113. [CrossRef]

17. Miralbes, R.; Castejon, L. Aerodynamic Analysis of Some Boat Tails for Heavy Vehicles. Int. J. Heavy Veh. Syst. 2012, 19, 115-127. [CrossRef]

18. Lee, E.J.; Lee, S.J. Drag Reduction of a Heavy Vehicle Using a Modified Boat Tail with Lower Inclined Air Deflector. J. Vis. 2017, 20, 743-752. [CrossRef]

19. Chowdhury, H.; Moria, H.; Ali, A.; Khan, I.; Alam, F.; Watkins, S. A Study on Aerodynamic Drag of a Semi-trailer Truck. Procedia Eng. 2013, 56, 201-205. [CrossRef]

20. Metka, M.; Gregory, J.W. Drag Reduction on the 25-deg Ahmed Model Using Fluidic Oscillators. J. Fluid Eng. 2015, 137, 051108. [CrossRef]

21. Hirz, M.; Stadler, S. A New Approach for the Reduction of Aerodynamic Drag of Long-Distance Transportation Vehicles; SAE Technical Paper 2013-01-2414; Graz University of Technology: Graz, Austria, 2013.

22. Kim, D.; Lee, H.; Yi, W.; Choi, H. A Bio-inspired Device for Drag Reduction on a three-dimensional Model Vehicle. Bioinspir. Biomim. 2016, 11. [CrossRef] [PubMed]

23. Kim, J.J.; Hong, J.; Lee, S.J. Bio-inspired Cab-roof Fairing of Heavy Vehicles for Enhancing Drag Reduction and driving Stability. Int. J. Mech. Sci. 2017, 131, 868-879. [CrossRef]

24. Hinglo, K.; Kontis, K. Flow Characteristics over a Heavy truck Model with and without Vane-type Vortex Generator Installed. J. Wind Eng. Ind. Aerodyn. 2016, 159, 110-122. [CrossRef]

25. Fukuchi, Y.; Sawada, J.; Nakajima, M.; Murakumo, Y. Development of a New Pressure Measurement Technique and PIV to Validate CFD for the Aerodynamics of Full-Scale Vehicles; SAE Technical Paper 2016-01-1623; Honda R\&D Co., Ltd.: Tokyo, Japan, 2016.

26. Landman, D.; Wood, R.; Seay, W.; Bledsoe, J. Understanding Practical Limits to Heavy Truck Drag Reduction; SAE Technical Paper 2009-01-2890; Old Dominion University: Norfolk, VA, USA, 2009.

27. Cooper, K.R. Wind Tunnel and Track Tests of Class 8 Tractors Pulling Single and Tandem Trailers Fitted with Side Skirts and Boat-Tails; SAE Technical Paper 2012-01-0104; National Research Council Canada: Ottawa, ON, Canada, 2012.

28. Lav, C. Three Dimensional CFD Analysis on Aerodynamic Drag Reduction of a Bluff Tractor Trailer Body Using Vortex Generators; SAE Technical Paper 2013-01-2458; Delhi Technological University: Delhi, India, 2013.

29. Fu, L. Automobile Design and Aerodynamics; China Machine Press: Beijing, China, 2011.

30. Kobayashi, T.; Kitoh, K. A Review of CFD Methods and Their Application to Automobile Aerodynamics; SAE Paper 920338; Kyushu University: Fukuoka, Japan, 1992.

31. Cheng, S.Y.; Tsubokura, M.; Nakashima, T.; Okada, Y.; Nouzawa, T. Numerical Quantification of Aerodynamic Damping on Pitching of Vehicle-inspired Bluff body. J. Fluids Struct. 2012, 30, 188-204. [CrossRef]

32. Yingchao, Z.; Wei, D.; Tao, C.; Shao, S. Aerodynamic Styling of Fairing for Commercial Vehicle Cab. Automot. Eng. 2014, 9, 1063-1067.

33. Menter, F.R. Two Equation Eddy-Viscosity Turbulence Models for Engineering Applications. AIAA J. 1994, 32, 1598-1605. [CrossRef]

34. Menter, F.R.; Kuntz, M.; Langtry, R. Ten Years of Industrial Experience with the SST Turbulence Model. Turbul. Heat Mass Transf. 2003, 4, 625-632.

35. Jia, Z. Numberical Simulation of Cars in the External Flow Field Based on Different Turbulence Models; Zheng Zhou University: Zheng Zhou, China, 2011.

(C) 2018 by the authors. Licensee MDPI, Basel, Switzerland. This article is an open access article distributed under the terms and conditions of the Creative Commons Attribution (CC BY) license (http://creativecommons.org/licenses/by/4.0/). 